\title{
African biogeographical ancestry, atopic and non-atopic asthma and atopy: A study in Latin American children
}

\author{
Thiago M. da Silva $\mathrm{PhD}^{1,2}$ (D) | Rosemeire L. Fiaccone $\mathrm{PhD}^{3,4}$ \\ Fernanda S. G. Kehdy PhD ${ }^{5}$ | Eduardo Tarazona-Santos PhD $^{6}$ । \\ Laura C. Rodrigues PhD ${ }^{7}$ | Gustavo N. O. Costa PhD ${ }^{1,4}$ | \\ Camila A. Figueiredo PhD ${ }^{8}$ | Darci N. dos Santos $\mathrm{PhD}^{1}$ | Caroline A. Feitosa PhD ${ }^{1}$ | \\ Gisel L. Fattore PhD ${ }^{1,9}$ | Leticia M. Santos PhD $^{1}$ | \\ Neuza M. Alcantara-Neves PhD ${ }^{8}$ | Álvaro A. Cruz PhD ${ }^{10}$ | Maurício L. Barreto PhD ${ }^{1,4}$ \\ 1 Institute of Collective Health, Federal University of Bahia, Salvador, Bahia, Brazil \\ 2 Departamento de Ciências Biológicas, Universidade Estadual do Sudoeste da Bahia, Jequié, Bahia, Brazil \\ ${ }^{3}$ Departamento de Estatística, Instituto de Matemática, Universidade Federal da Bahia, Salvador, Bahia, Brazil \\ ${ }^{4}$ Center of Data and Knowledge Integration for Health, Instituto Gonçalo Muniz, Fundação Osvaldo Cruz, Salvador, Brazil \\ ${ }^{5}$ Laboratório de Hanseníase, Instituto Oswaldo Cruz, Fundação Oswaldo Cruz, Rio de, Janeiro, Brazil \\ ${ }^{6}$ Departamento de Biologia Geral, Instituto de Ciências Biológicas, Universidade Federal de Minas Gerais, Belo Horizonte, Minas Gerais, Brazil \\ 7 Department of Infectious Disease Epidemiology, Faculty of Epidemiology, London School of Hygiene and Tropical Medicine, London, United Kingdom \\ 8 Instituto de Ciências da Saúde, Universidade Federal da Bahia, Salvador, Bahia, Brazil \\ 9 Departamento de Salud Comunitaria, Universidad Nacional de Lanús, Buenos Aires, Argentina \\ 10 ProAR-Center of Excellence in Asthma, Federal University of Bahia School of Medicine, Salvador, Brazil
}

\section{Correspondence}

Maurício Lima Barreto, PhD, Center of Data and Knowledge Integration for Health, Fundação Oswaldo Cruz, Parque Tecnológico da Bahia, Rua Mundo, 121, Trobogy, Salvador - Bahia, 41745-715 Salvador - Ba, Brazil.

Email: mauricio.barreto@bahia.fiocruz.br

Funding information

Department of Science and Technology (DECIT, Ministry of Health); National Fund for Scientific and Technological Development (FNDCT, Ministry of Science and Technology); (FINEP, Ministry of Science and Technology, Brazil)

\section{Abstract}

Background: Genetic variants underlying African ancestry have been suggested be implicated in the ethnic-racial inequalities reported for asthma and allergies.

Objectives: To investigate the association between individual African ancestry and asthma symptoms, atopic and non-atopic asthma, and atopy in children.

Methods: A cross-sectional study encompassing 1190 individuals was conducted. African biogeographic ancestry was estimated using 370539 genome-wide SNPs. Serum levels of specific IgE were measured, and skin prick test (SPT) performed for the most common local aeroallergens. Information on asthma symptoms was obtained by applying the International Study of Allergy and Asthma in Childhood questionnaire. The associations between the proportion of individual African ancestry and the outcomes investigated were analyzed through multivariate models adjusted for socio-environmental variables, infections markers, and psychosocial factors.

Results: Each $20 \%$ increase in the proportion of African ancestry was negatively associated with SPT reactivity (OR: $0.79,95 \% \mathrm{Cl}: 0.66-0.96)$ and positively associated with asthma symptoms in non-atopic individuals (OR: 1.40, 95\% Cl: 1.03-1.89). We estimated that socioeconomic status and number of infections mediated $28.4 \%$ of the effect of African ancestry on SPT reactivity, while $20.2 \%$ of the effect on 
non-atopic asthma was explained by socioeconomic status and behavioral problems in children.

Conclusions: The negative association observed between African ancestry and atopy is most probably explained by unobserved environmental or social factors that covariate with ancestry. For non-atopic asthma, in turn, putative genetic variants of risk underlying African ancestry may play some role.

\section{KEYWORDS}

allergy, asthma, biogeographical ancestry, mediation, racial inequalities

\section{1 | INTRODUCTION}

Ethnic-racial inequalities for asthma and atopy have been extensively reported. ${ }^{1,2}$ Interestingly, although different studies indicate that the prevalence of asthma and allergies is higher in individuals of African ancestry living in developed countries, a lower prevalence of such outcomes is observed in African countries. ${ }^{3}$ An explanatory hypothesis for these findings is that individuals with predominant African ancestry would have a higher frequency of pro-inflammatory genetic variants as consequence of the evolutionary adaptation of African ancestors to an environment with high infection burden, especially helminth infections. ${ }^{4}$ Reducing exposure to pathogens due to better hygienic conditions and westernized lifestyle would make these pro-inflammatory genetic variants act as risk factors for asthma and allergies. ${ }^{5}$ This hypothesis is supported by studies that indicate that pathogen infections were one of the major forces that shaped the genetic makeup of current human populations. ${ }^{6}$ Also, several genes undergone to natural selection by helminth infections are also involved in susceptibility to asthma. ${ }^{7}$ Finally, different studies have reported a higher risk for asthma and atopy with increasing proportion of individual African ancestry. ${ }^{8-10}$

Importantly, biogeographic ancestry also captures socioeconomic, environmental, and psychosocial factors that are unevenly distributed among ethnic/racial groups and that affect the health status of individuals. ${ }^{11}$ Thus, studies that aim to study the association between biogeographic ancestry and asthma and atopy should take into account the influence of these important non-genetic determinants. In this aspect, the SCAALA-Salvador project ${ }^{12}$ occupies a privileged position, having a range of information on socioeconomic status, environmental variables, infection markers, and psychosocial factors in a cohort of a large urban center in northeastern Brazil and with one of the highest prevalence of asthma symptoms worldwide. ${ }^{13}$

Although the association between African ancestry and asthma has been previously reported, the relationship of African ancestry with atopic and non-atopic asthma has not been studied to date. This investigation is important since the risk factors and immunological mechanisms involved in atopic and non-atopic asthma are probably different from each other. ${ }^{14}$ Furthermore, to our knowledge, no study to date assessed the effect of African ancestry on asthma and atopy mediated by socioeconomic, environmental, and psychosocial factors.
Since lower socioeconomic status and indicator variables of poorer environmental and psychosocial conditions affect the risk of atopy and asthma in our population, ${ }^{15-17}$ it is reasonable to suppose that part of the effect of African ancestry on these outcomes may be mediated by such factors.

Thus, in the present study, we investigated the association of African ancestry with asthma symptoms, atopic and non-atopic asthma, and atopy in an urban and highly mixed population in Latin America. Also, we estimated the effect of African ancestry on these different outcomes mediated by socioeconomic status (family income and maternal schooling), infection burden, and psychosocial factors (behavioral problems in children).

\section{2 | METHODS}

\section{1 | Study population}

A cross-sectional study involving 1190 children enrolled in the SCAALA program (Social Change in Asthma and Allergies in Latin America) in the city of Salvador (State of Bahia, Brazil). The study population comprises a cohort recruited from poor neighborhoods of the city, originally accompanied to assess the impact of a sanitation program on health conditions in childhood. ${ }^{12}$ This first study followed three separate cohorts of children aged 0-3 years recruited from 24 small geographical areas selected to represent the population without sanitation in Salvador, called sentinel-areas. ${ }^{18}$ These cohorts were recruited in 1997, 2001, and 2003, respectively, totaling about 3000 children. A random selection of children living in sentinel-areas was performed during the recruitment of each of the three cohorts. The remaining children of these three original cohorts who were aged 4-11 years in the year 2005 and had complete follow-up information $(n=1,445)$ were selected for the SCAALA-Salvador cohort. The data used in the present study were obtained through questionnaires applied to the parents or guardians between 1997 and 2006, and the outcomes and most of the variables were collected in 2005 when the children were between 4 and 11 years of age. The free and informed consent form was signed by the parents or guardians of each of the children, and the study was approved by the Research Ethics Committee of the UFBA Institute of Public Health, under registry number 003-05/CEP-ISC. 


\section{2 | Instruments and data collection}

A questionnaire translated and adapted from the International Study of Asthma and Allergy in Childhood (ISAAC) which has been validated internationally as well in Brazil ${ }^{12}$ was used to identify asthma symptoms, family history of allergies, exposure to allergens, and presence of smokers in the home.

Psychosocial factors were measured by the application of different internationally validated instruments. More details on the instruments used and the criteria applied for the classification of individuals are given in the supplementary Methods section (see online data supplement).

A questionnaire was applied to assess variables such as maternal schooling, family income, basic sanitation, and housing conditions, exposure to dirt and demographic characteristics. Infection (or pathogen exposure) was defined by the presence of positive results in serological tests for seven pathogens (Toxocara spp, Toxoplasma gondii, Helicobacter pylori, and hepatitis A, herpes simplex, herpes zoster, and Epstein-Barr viruses) and the presence of intestinal helminth eggs in stool samples (Ascaris lumbricoides and Trichiuris trichiura). A threshold of three infections or less was used to distinguish light from heavy infection, as previously reported. ${ }^{15}$ Details about the anthropometric measurements are given in the supplementary Methods section (see online data supplement).

\subsection{Genotyping and estimative of African ancestry}

The proportion of individual African ancestry was estimated using ADMIXTURE software. Briefly, a tri-hybrid model was used in the unsupervised analyses performed in ADMIXTURE. ${ }^{19}$ As external panels samples of African and European individuals from the HapMap project and 93 Native American of the Human Genome Diversity (HGDP) project were used. A total of 370539 SNPs shared by samples from the HapMap, HGDP and the study population were used for biogeographical ancestry estimate. More details on genotyping and biogeographical ancestry estimate are given in the supplementary Methods section in the online data supplement.

\section{$2.4 \mid$ Outcomes and covariates}

The occurrence of asthma symptoms in children was defined as the presence of wheezing in the last 12 months in addition to one of the following conditions: clinical diagnosis of asthma ever, wheezing after exercise in the last 12 months, 4 or more episodes of wheezing in the last 12 months, and wake up at night because of wheezing in the last 12 months.

Skin prick tests (SPTs) were done with seven common aeroallergens (Dermatophagoides pteronyssinus, Blomia tropicalis, Blattella germanica, Periplaneta americana, fungi, and cat and dog dander). Saline and $10 \mathrm{mg} / \mathrm{mL}$ histamine solution were used as negative and positive controls, respectively. Reactions were read after $15 \mathrm{~min}$, and a mean wheal size of at least $3 \mathrm{~mm}$ greater than that elicited by the negative control was considered positive. Determination of specific
IgE serum concentrations was performed for four allergens (D. pteronyssinus, B. tropicalis, B. germanica, and P. americana). Children with $0.70 \mathrm{kU} / \mathrm{L}$ or greater of specific IgE for any allergen tested were considered to have positive results.

Atopic and non-atopic asthma were defined by the presence of asthma symptoms, as defined above, in the presence or absence, respectively, of a positive result for SPT and/or specific IgE for at least one of the aeroallergens tested.

The covariates considered in the present study were: sex and age of the child, maternal schooling, family income, birth weight, BMI Z-scores, exposure to smoke in the home, attendance at day care center, reported presence of rodents at home, exposure to mold at home, street paving, presence of tap water, infection burden, $T$. trichiura infection up to 3 years of age, racial discrimination reported by parents or guardians, minor psychiatric disorders in parents or guardians, and behavioral problems in children. Most of these variables have been associated with asthma and atopy in different studies and are also potentially associated with the proportion of individual African ancestry.

\section{5 | Statistical analysis}

The associations between the proportion of individual African ancestry and asthma symptoms, SPT, and specific IgE for aeroallergens were analyzed using multivariate binary logistic regression models. The association of African ancestry with asthma atopic and non-atopic asthma was analyzed by multivariate polytomic logistic regression, as previously described. ${ }^{16}$ The variables nominally associated with the investigated outcomes were introduced in the multivariate models using the backward method, and the variables statistically significant remained in the final models. Variables usually referred in the literature of asthma and atopy as potential confounders, in addition to sex and age, were also maintained in the final models, regardless of statistical significance. Odds Ratio (OR) measures and their respective $95 \%$ confidence intervals were calculated to measure the chance of the investigated outcomes for each $20 \%$ increase in the proportion of individual African ancestry. Mediation analyses were performed in the SPSS 20.0 (IBM Corp., Armonk, NY) PROCESS module ${ }^{20}$ to evaluate the effect of African ancestry on SPT explained by socioeconomic factors and infection burden, as well the effect of African ancestry on non-atopic asthma explained by socioeconomic status and behavioral problems in children. All statistical analyses were performed on Stata version 12.0 (Stata Corporation, College Station, TX). Since the different outcomes evaluated are not independent of each other, no correction for multiple tests was performed.

\section{3 | RESULTS}

The selection criteria of the study population are shown in Figure 1. Except for sex, rodents in home and tap water, no significant differences were observed between children included and excluded from the study (Table S1 in online data supplement). The prevalence 


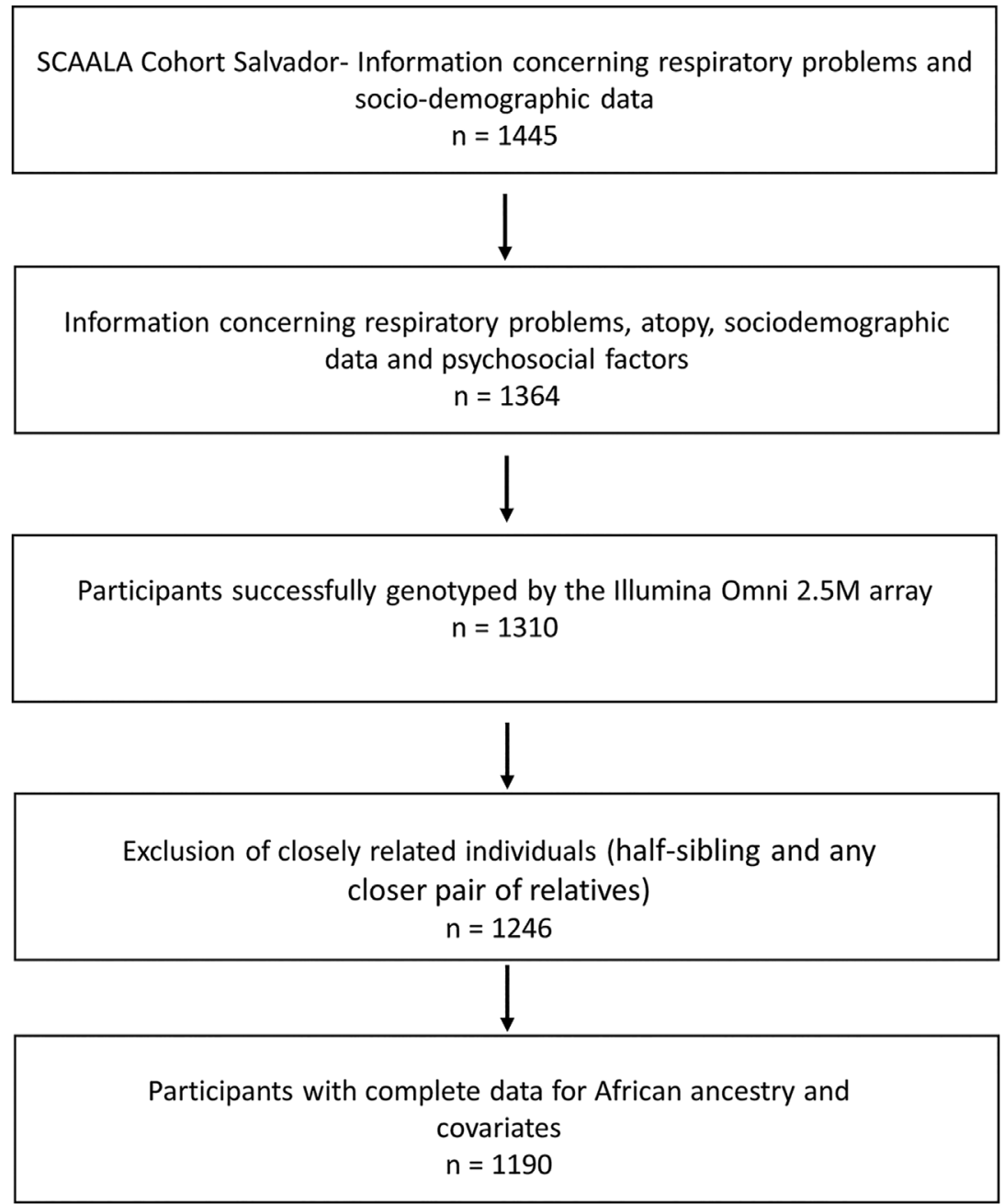

FIGURE 1 Selection of study population

of investigated outcomes are shown in Table S2 and the demographic and clinical characteristics of the different groups compared in the study are shown in Table S3 (online data supplement). The median of African ancestry in the general population was $50.59 \%$ (interquartile range: $41.0-60.25 \%$ ). The distribution of the proportion of individual African ancestry in the study population is shown in Supplementary Figure S1.

The results of the association between the proportion of individual African ancestry and the different outcomes investigated are presented in Table 1. In the models adjusted for the sex and age the

TABLE 1 Odds Ratio of slgE, SPT, atopy and asthma symptoms according to the increased proportion of individual African ancestry ( $n=1190$ )

\begin{tabular}{|c|c|c|c|c|}
\hline Outcomes & Cases/Non-cases & Model 1 OR $(95 \% \mathrm{Cl})$ & Model 2 OR $(95 \% \mathrm{Cl})$ & Model 3 OR (95\%Cl) \\
\hline slgE $>0.70$ & $451 / 739$ & & & \\
\hline$\%$ African ancestry & & $0.84(0.70-0.99) P=0.047$ & $0.87(0.73-1.04) P=0.086$ & - \\
\hline$\%$ African ancestry & & $0.71(0.59-0.85) \mathrm{P}<0.001$ & $0.79(0.66-0.96) P=0.005$ & - \\
\hline Atopy (slgE + SPT +) & $512 / 678$ & & & \\
\hline Asthma symptoms & $272 / 918$ & & & \\
\hline$\%$ African ancestry & & $1.23(1.01-1.49) P=0.042$ & - & $1.18(0.95-1.45) P=0.130$ \\
\hline
\end{tabular}

Model 1: adjusted by sex and age. Model 2: adjusted by sex, age, family income, infection burden, $T$. trichiuri infection up to 3 years, attendance at day care, presence of rodents at home, tap water and street paving. Model 3: adjusted by gender, age, mother education, smoke in the home, birth weight, BMI Z-scores, minor psychiatric disorders in parents and behavioral problems in children 
increase of the proportion of individual African ancestry was negatively associated with specific $\lg \mathrm{E}>0.70 \mathrm{kU} / \mathrm{L}$ for at least one aeroallergen (OR: $0.84,95 \% \mathrm{Cl}$ : 0.70-0.99), SPT positive for at least one aeroallergen (OR: $0.71,95 \% \mathrm{Cl}: 0.59-0.85$ ), and positivity for at least one of these allergy markers (OR: $0.80,95 \% \mathrm{Cl}$ : $0.68-0.95)$. A positive association, on the other hand, was observed between the proportion of African ancestry and asthma symptoms (OR: 1.23 ; $95 \% \mathrm{Cl}: 1.01-$ 1.49). In the analyzes adjusted for potential confounders of the association between African ancestry and these outcomes, however, only the results for positive SPT (OR: $0.79,95 \% \mathrm{Cl}: 0.66-0.96)$ and positivity for slgE and SPT (OR: $0.84,95 \% \mathrm{Cl}$ : 0.71-0.99) remained statistically significant. For asthma symptoms (OR: 1.18, 95\%Cl: $0.95-$ 1.45) and slgE (OR: $0.87,95 \% \mathrm{Cl}: 0.73-1.04)$ the associations lacked statistical significance. These results were practically unchanged in the models that retained only the potential confounders statistically associated with the considered outcomes (data not shown).

Table 2 shows the results of the polytomic regression for the atopic and non-atopic asthma. In the adjusted analyzes for sex and age the increase in the proportion of African ancestry was positively associated with asthma symptoms in non-atopic individuals (OR: 1.47; 95\% Cl: $1.10-1.95)$. These associations remained statistically significant after adjusting for potential confounders (OR: 1.40; 95\%Cl: 1.031.89). On the other hand, no significant association was observed between individual African ancestry and asthma symptoms in atopic individuals.

Mediation analyzes were performed to quantify the proportion of the total effect of African ancestry on SPT mediated by family monthly income and number of infections (Figure 2A), as well as the proportion of the total effect of African ancestry on non-atopic asthma mediated through maternal education and behavioral problems in children (Figure 2B). In both models, proxy variables for socioeconomic status are considered as distal causes (or fundamental causes) that originate the proximal causes represented by infection burden (for SPT reactivity outcome) and behavioral problems in children (for nonatopic asthma outcome). ${ }^{21}$ The choice of the mediators for each of the conceptual models tested was based on the reported effect of these variables on both SPT reactivity and non-atopic asthma in studies conducted in our population and elsewhere. ${ }^{14,17}$ As shown in Table 3, family income and number of infections mediated $28.4 \%$ of the effect of African ancestry on SPT reactivity. For non-atopic asthma, in turn, a lower proportion of the indirect effect was observed, with only $20.2 \%$ of the total effect of African ancestry being explained by maternal schooling and behavioral problems in children.

\section{4 | DISCUSSION}

In the present study, we report a negative association between individual African ancestry and atopy, and we also describe for the first time the association between African ancestry and atopic and nonatopic asthma, observing a positive relation with non-atopic asthma. To our knowledge, this is the largest and one of the first studies to investigate the association between African ancestry and asthma symptoms, atopic and non-atopic asthma and atopy in a Latin American population.

Positive associations between individual African ancestry and asthma independent of atopy status have previously been reported for African-American, ${ }^{8,22}$ Caribbean, ${ }^{10,23}$ and US Latino populations. ${ }^{9}$ In our population, in models adjusted for sex and age only, a positive association between African ancestry and asthma symptoms was also observed. However, this association lacked significance in multivariate models, which is in agreement with that reported by other authors. ${ }^{24}$ It is noteworthy, however, that African ancestry was independently associated with the risk of asthma in studies that considered the influence of socioeconomic status ${ }^{10}$ as well as variables related to several environmental exposures in prenatal and early childhood. ${ }^{22}$ Also, in the largest study conducted so far and that has taken into account the influence of socioeconomic, environmental, and psychosocial factors, African ancestry was positively and significantly associated with asthma. ${ }^{9}$ These results suggest that part of this association may be explained by underlying genetic factors, although residual confounding should not be completely ruled out.

The association between individual African ancestry and atopy has been less extensively investigated, but some results were previously reported. A positive association with allergen-specific IgE has been described among asthmatics from an admixed population in the Canary Islands. ${ }^{24}$ Positive associations between the proportion of African ancestry and total IgE were also reported for Caribbean populations. ${ }^{10,23}$ However in regions where helminthiasis is endemic the usefulness of total IgE as a marker of allergies is limited, ${ }^{25}$ and these studies do not provide estimates adjusted for helminth infections. Kumar et $\mathrm{al}^{26}$ in turn, reported a positive association of African ancestry with specific IgE for food allergens in an ethnically heterogeneous United State population, which remained significant after adjustments for potential confounders. In the present study, however, we found an inverse association between the proportion of individual African ancestry and atopy, especially when measured by SPT reactivity. These results are in disagreement with the hypothesis

TABLE 2 Odds Ratio of non-atopic asthma and atopic asthma according to the increased proportion of individual African ancestry $(n=1190)$

\begin{tabular}{llll}
\hline Asthma phenotypes & Cases/Non-cases & Model 1 OR $(95 \% \mathrm{Cl})$ & Model 2 OR (95\%Cl) \\
\hline $\begin{array}{l}\text { Non-atopic asthma (vs non-atopic and non-asthmatic) } \\
\text { \% African ancestry }\end{array}$ & $129 / 549$ & $\mathbf{1 . 4 7}(1.10-1.95) P=0.008$ & $1.40(1.03-1.89) P=0.031$ \\
\hline $\begin{array}{l}\text { Atopic asthma (vs atopic and non asthmatic) } \\
\text { \% African ancestry }\end{array}$ & $143 / 369$ & $1.11(0.84-1.46) P=0.463$ & $1.07(0.80-1.43) P=0.656$
\end{tabular}

Model 1: adjusted by sex and age. Model 2: adjusted by sex, gender, age, maternal education, presence of mold at home, smoke in the home, birth weight, BMI Z-scores, minor psychiatric disorders in parents, behavioral problems in children and attendance at day care 

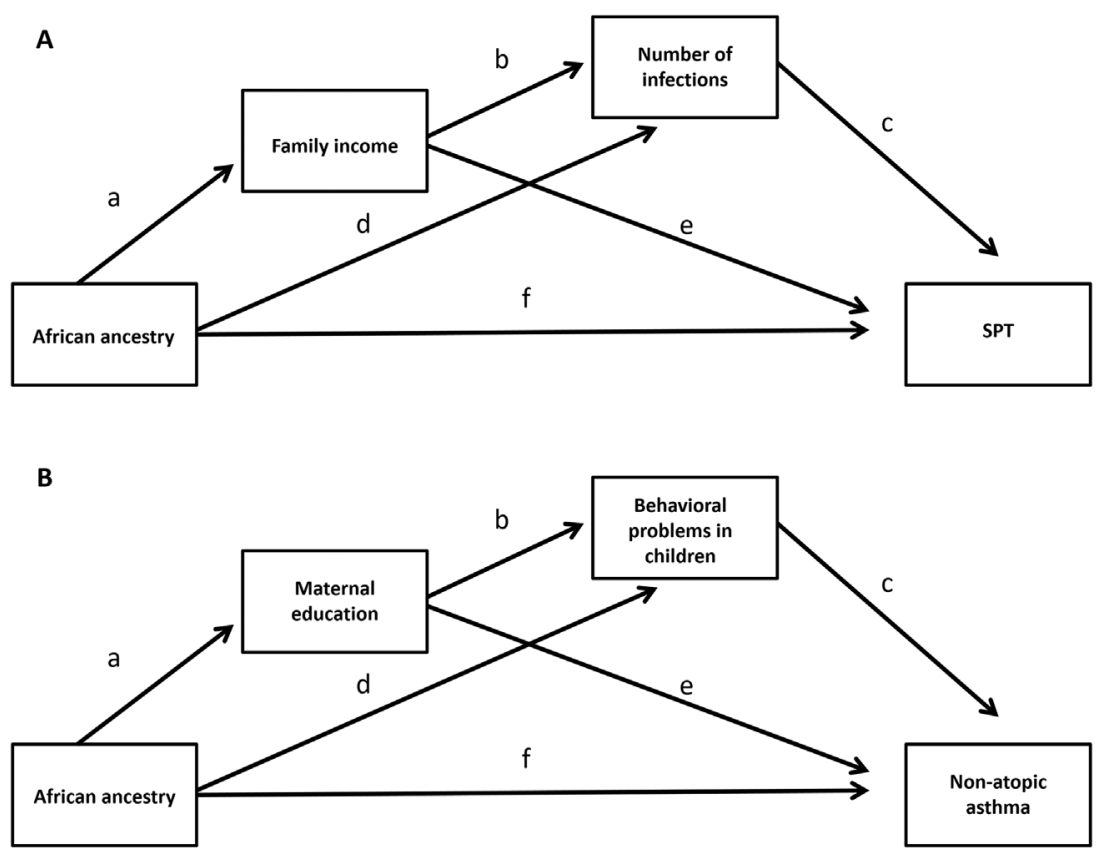

FIGURE 2 Conceptual models for SPT reactivity (A) and non-atopic asthma (B). Indirect effect: abc + dc + ae; Direct effect: f; Total effect: $a b c+d c+a e+f$

that African ancestry could act as a proxy for pro-inflammatory genetic variants which would predispose to development of allergic symptoms. $^{4}$

Furthermore, although in our population the association between African ancestry and atopy has remained significant in multivariate models, the direction of this association is more likely to be explained by the influence of unmeasured environmental or social exposures that covariate with ancestry. It has been shown, for instance, that both lower socioeconomic status and exposure to poorer hygienic-sanitary conditions, factors that are directly related to the proportion of African ancestry, are negatively associated with atopy. ${ }^{15,27}$ Indeed, in mediation analyses, we estimated that family income and infection burden mediated the effect of African ancestry on SPT reactivity in almost $30 \%$. Also, some authors have suggested that location of residence may be a proxy for environmental variables capable of

TABLE 3 Total and indirect effect of individual African ancestry on SPT and non-atopic asthma in the study population

\begin{tabular}{|l|l|}
\hline & \% African ancestry \\
\cline { 2 - 2 } Effect size & Coeficient $(95 \% \mathrm{Cl})$ \\
\hline SPT & \\
\hline \multicolumn{1}{l}{ Total effect } & $-1.738(-2.644--0.831)$ \\
\hline \multicolumn{1}{l}{ Indirect effect } & $-0.493(-0.750--0.286)$ \\
\hline Indirect effect/Total effect (\%) & 28.4 \\
\hline Non-atopic asthma & \\
\hline Total effect & $2.091(0.592-3.591)$ \\
\hline Indirect effect & $0.422(0.084-0.759)$ \\
\hline Indirect effect/Total effect (\%) & 20.2 \\
\hline
\end{tabular}

overcoming the possible influence of risk genetic factors for atopy underlying African ancestry. ${ }^{28}$

To our knowledge, this is the first study to evaluate the association between individual African ancestry and atopic and non-atopic asthma. Our results indicate that African ancestry is more relevant for non-atopic asthma, with no association being verified for atopic asthma. This is not surprising when we consider the relative dissociation between atopy and asthma symptoms, especially in Latin America, where only a small fraction of the population prevalence of asthma is attributable to atopy. ${ }^{29}$ Also, these results are consistent with the observation that in our population the main risk factors associated with non-atopic asthma are those related to poverty, exposure to dirt, infections in early childhood, and psychosocial problems, all potentially associated with individual African ancestry. ${ }^{16,17}$ Indeed, the association between lower socioeconomic status and non-atopic asthma has been reported for other populations. ${ }^{30}$

However, unlike the observed association of African ancestry with asthma symptoms in general, the association with non-atopic asthma remained significant in multivariate analyzes. Also, mediation analyses demonstrated that maternal education and behavioral problems in children mediated only $20 \%$ of the effect of African ancestry on asthma symptoms in non-atopic individuals. Although the presence of residual confounding should not be ruled out, these results may also suggest the existence of a more complex pattern between African ancestry and non-atopic asthma.

In this sense, the putative proinflammatory genetic predisposition in individuals with a higher proportion of African ancestry, which would also involve genes related to innate immunity, ${ }^{31}$ would be eliceted by environmental factors that would lead to the development of non-allergic airway inflammation. An example would be exposure to 
airway irritants, such as endotoxins and particulate matter from pollution, which would be more common in individuals subjected to poorer living conditions and housing, especially in large urban centers in Latin America. ${ }^{32}$ Such compounds could act as triggers for inflammatory processes that would result in the development of non-atopic asthma, ${ }^{33}$ with a higher risk in those individuals with a more pro-inflammatory immune response profile. Environmental triggers of another nature but also linked to lower socioeconomic status, such as exposure to gun violence, could also interact with African ancestry in the risk of asthma, as recently reported. ${ }^{34}$ This hypothesis is supported by the observation made in a large study that African Americans children were at higher asthma risk than European Americans children only among that very poor. ${ }^{35}$ This suggests that genetic factors underlying African ethnicity would be more important for the development of asthma in low-income and underprivileged populations, such as the analyzed in the present study. However, this hypothesis needs to be further explored in larger studies and with more power to perform interaction analyzes between biogeographic ancestry and socioeconomic status for asthma risk, especially non-atopic asthma. Alternatively, nutritional factors such as vitamin $D$ deficiency, which is more common in individuals of African origin, $^{36}$ may also explain the observed results. Indeed, different observational studies and clinical trials have demonstrated the role of vitamin D in asthma morbidity, ${ }^{37}$ and vitamin D supplementation has been associated with reduced eosinophilic airway inflammation in patients with nonatopic asthma. ${ }^{38}$

The present study has some limitations. First, the diagnosis of asthma was based on self-reported information. Nevertheless, we have tried to minimize eventual bias by using more accurate definition for asthma (presence of wheezing in the last 12 months plus symptoms). However, the likely heterogeneity resulting from non-use of clinical criteria for the definition of asthma cases may have contributed to the lack of study power. Second, asthma is difficult to diagnose before age 6 years, and a considerable portion of the children evaluated were aged between 4 and 5 years. Indeed, when analyzes were restricted to children older than 5 years, the association of African ancestry with non-atopic asthma lacked statistical significance (Table S3 in online data supplement). Interestingly, in children aged 5 years or less this association remained significant, which may suggest a modifying effect of age (Table S4 in online data supplement). On the other hand, the association observed in younger children may reflect a greater influence of African ancestry on transient wheezing, which has not been related to an atopic predisposition. ${ }^{39}$ However, this needs to be confirmed in further studies. Third, because it was a cross-sectional study, the time-dependent effects of some exposures (such as infection burden and behavioral problems) could not be adequately measured. Fourth, although a correction for multiple tests has not been adopted because of the dependence between the different outcomes analyzed (for instance: asthma symptoms and atopy), the possibility of some results being due to chance should not be rules out. Finally, due to relatively small sample size, the study lacks power mainly for the analysis of association between individual African ancestry and asthma symptoms in atopic and non-atopic individuals, which suggests caution in the interpretation of the results presented here. Indeed, for both atopic as well as non-atopic asthma the study shows a power of $45 \%$ only to detect a modest effect ( $O R=1.20$ ) according to the continuous variation of African ancestry (Table S5 in online data supplement).

In summary, we found that individual African ancestry was negatively associated with atopy and positively associated with non-atopic asthma in a highly mixed and low-income urban population of Latin America. Although these results have remained significant in multivariate analysis, the pattern of association of African ancestry with these outcomes mimics that observed for the lower socioeconomic status. However, while the findings for atopy indicate that possible genetic factors underlying African ancestry are likely less important than non-genetic determinants, in the case of non-atopic asthma such genetic variants may play some role, especially in interaction with environmental factors. Further studies will be needed to clarify these complex relationships between biogeographical ancestry, atopic and non-atopic asthma, and atopy.

\section{ACKNOWLEDGMENTS}

This work was supported by the Department of Science and Technology (DECIT, Ministry of Health) and National Fund for Scientific and Technological Development (FNDCT, Ministry of Science and Technology), Funding of Studies and Projects (FINEP, Ministry of Science and Technology, Brazil).

\section{AUTHORS' CONTRIBUTIONS}

TMS, RLF, and MLB conceived the study. MLB is the cohort coordinator, providing samples and data. FSGK and ET-S coordinated the genomic analyses. TMS wrote the manuscript. All the authors contributed with discussion on the results and on the manuscript. The authors GNOC, LCR, CAF, DNS, CAF, GLF, LMS, NMA-N, and AAC contributed with data, bioinformatic resources or statistical analyses.

\section{CONFLICTS OF INTEREST}

The authors declare no conflict of interest.

\section{ORCID}

Thiago M. da Silva iD http://orcid.org/0000-0001-5171-0123

\section{REFERENCES}

1. Lester LA, Rich SS, Blumenthal MN, et al. Ethnic differences in asthma and associated phenotypes: collaborative study on the genetics of asthma. J Allergy Clin Immunol. 2001;108: 357-362.

2. Panico L, Bartley M, Marmot M, Nazroo JY, Sacker A, Kelly YJ. Ethnic variation in childhood asthma and wheezing illnesses: findings from the Millennium Cohort Study. Int J Epidemiol. 2007;36: 1093-1102.

3. Obeng BB, Hartgers F, Boakye D, Yazdanbakhsh M. Out of Africa: what can be learned from the studies of allergic disorders in Africa and Africans? Curr Opin Allergy Clin Immunol. 2008;8: 391-397. 
4. Le Souef PN, Candelaria P, Goldblatt J. Evolution and respiratory genetics. Eur Respir J. 2006;28: 1258-1263.

5. Rook GA. Hygiene and other early childhood influences on the subsequent function of the immune system. Dig Dis. 2011;29: 144-153.

6. Fumagalli M, Sironi M, Pozzoli U, Ferrer-Admetlla A, Pattini L, Nielsen R. Signatures of environmental genetic adaptation pinpoint pathogens as the main selective pressure through human evolution. PLoS Genet. 2011;7: e1002355.

7. Fumagalli M, Pozzoli U, Cagliani R, et al. The landscape of human genes involved in the immune response to parasitic worms. BMC Evol Biol. 2010;10:264.

8. Flores C, Ma SF, Pino-Yanes M, et al. African ancestry is associated with asthma risk in African Americans. PLoS ONE. 2012;7: e26807.

9. Pino-Yanes M, Thakur N, Gignoux CR, et al. Genetic ancestry influences asthma susceptibility and lung function among Latinos. J Allergy Clin Immunol. 2015;135: 228-235.

10. Vergara C, Caraballo L, Mercado D, et al. African ancestry is associated with risk of asthma and high total serum IgE in a population from the Caribbean Coast of Colombia. Hum Genet. 2009;125: 565-579.

11. Parra EJ, Kittles RA, Shriver MD. Implications of correlations between skin color and genetic ancestry for biomedical research. Nat Genet. 2004;36: S54-S60.

12. Barreto ML, Cunha SS, Alcantara-Neves N, et al. Risk factors and immunological pathways for asthma and other allergic diseases in children: background and methodology of a longitudinal study in a large urban center in Northeastern Brazil (Salvador-SCAALA study). BMC Pulm Med. 2006;6:15.

13. Asher MI, Montefort S, Bjorksten B, et al. Worldwide time trends in the prevalence of symptoms of asthma, allergic rhinoconjunctivitis, and eczema in childhood: ISAAC Phases One and Three repeat multicountry cross-sectional surveys. Lancet. 2006;368: 733-743.

14. Strina A, Barreto ML, Cooper PJ, Rodrigues LC. Risk factors for nonatopic asthma/wheeze in children and adolescents: a systematic review. Emerg Themes Epidemiol. 2014;11:5.

15. Alcantara-Neves NM, Veiga RV, Dattoli VC, et al. The effect of single and multiple infections on atopy and wheezing in children. J Allergy Clin Immunol. 2012;129: 359-367. 367 e351-353.

16. Barreto ML, Cunha SS, Fiaccone R, et al. Poverty, dirt, infections and non-atopic wheezing in children from a Brazilian urban center. Respir Res 2010;11:167.

17. Feitosa CA, Santos DN, Barreto do Carmo MB, et al. Behavior problems and prevalence of asthma symptoms among Brazilian children. J Psychosom Res. 2011;71: 160-165.

18. Teixeira Md Mda G, Barreto ML, Costa Mda C, Strina A, Martins D, Jr., Prado M. Sentinel areas: a monitoring strategy in public health. Cad Saude Publica. 2002;18: 1189-1195.

19. Alexander $\mathrm{DH}$, Novembre J, Lange K. Fast model-based estimation of ancestry in unrelated individuals. Genome Res. 2009;19: 1655-1664.

20. Hayes AF, Preacher KJ. Statistical mediation analysis with a multicategorical independent variable. Br J Math Stat Psychol. 2014;67: 451-470.

21. Link BG, Phelan J. Social conditions as fundamental causes of disease. $J$ Health Soc Behav. 1995;Spec No:80-94.

22. Kumar R, Tsai HJ, Hong X, et al. African ancestry, early life exposures, and respiratory morbidity in early childhood. Clin Exp Allergy. 2012;42: 265-274.

23. Vergara C, Murray T, Rafaels N, et al. African ancestry is a risk factor for asthma and high total IgE levels in African admixed populations. Genet Epidemiol. 2013;37: 393-401.

24. Pino-Yanes M, Corrales A, Cumplido J, et al. No association between genetic ancestry and susceptibility to asthma or atopy in Canary Islanders. Immunogenetics. 2012;64: 705-711.
25. Arruda LK, Santos AB. Immunologic responses to common antigens in helminthic infections and allergic disease. Curr Opin Allergy Clin Immunol. 2005;5: 399-402.

26. Kumar R, Tsai HJ, Hong X, et al. Race, ancestry, and development of food-allergen sensitization in early childhood. Pediatrics. 2011;128: e821-e829.

27. Uphoff E, Cabieses B, Pinart M, Valdes M, Anto JM, Wright J. A systematic review of socioeconomic position in relation to asthma and allergic diseases. Eur Respir J. 2015;46: 364-374.

28. Kumar R, Nguyen EA, Roth LA, et al. Factors associated with degree of atopy in Latino children in a nationwide pediatric sample: the Genesenvironments and Admixture in Latino Asthmatics (GALA II) study. J Allergy Clin Immunol 2013;132: 896-905. e891.

29. Moncayo AL, Vaca M, Oviedo G, et al. Risk factors for atopic and nonatopic asthma in a rural area of Ecuador. Thorax. 2010;65: 409-416.

30. Court CS, Cook DG, Strachan DP. Comparative epidemiology of atopic and non-atopic wheeze and diagnosed asthma in a national sample of English adults. Thorax. 2002;57: 951-957.

31. Hagel I, Di Prisco MC, Goldblatt J, Le Souef PN. The role of parasites in genetic susceptibility to allergy: IgE, helminthic infection and allergy, and the evolution of the human immune system. Clin Rev Allergy Immunol. 2004;26: 75-83.

32. Mallol J, Castro-Rodriguez JA, Cortez E, Aguirre V, Aguilar P, Barrueto L. Heightened bronchial hyperresponsiveness in the absence of heightened atopy in children with current wheezing and low income status. Thorax. 2008;63: 167-171.

33. Drews AC, Pizzichini MM, Pizzichini E, et al. Neutrophilic airway inflammation is a main feature of induced sputum in nonatopic asthmatic children. Allergy. 2009;64: 1597-1601.

34. Rosas-Salazar C, Han YY, Brehm JM, et al. Gun violence, African ancestry, and asthma: a case-Control study in Puerto Rican children. Chest 2016;149: 1436-1444.

35. Smith LA, Hatcher-Ross JL, Wertheimer R, Kahn RS. Rethinking race/ ethnicity, income, and childhood asthma: racial/ethnic disparities concentrated among the very poor. Public Health Rep. 2005;120: 109-116.

36. Paul G, Brehm JM, Alcorn JF, Holguin F, Aujla SJ, Celedon JC. Vitamin D and asthma. Am J Respir Crit Care Med. 2012;185: 124-132.

37. Jolliffe DA, Greenberg L, Hooper RL, et al. Vitamin D supplementation to prevent asthma exacerbations: a systematic review and meta-analysis of individual participant data. Lancet Respir Med 2017;5: 881-890.

38. de Groot JC, van Roon EN, Storm H, et al. Vitamin D reduces eosinophilic airway inflammation in nonatopic asthma. J Allergy Clin Immunol. 2015;135: 670-675. e673.

39. Simon MR, Havstad SL, Wegienka GR, Ownby DR, Johnson CC. Risk factors associated with transient wheezing in young children. Allergy Asthma Proc. 2008;29: 161-165.

\section{SUPPORTING INFORMATION}

Additional supporting information may be found online in the Supporting Information section at the end of the article.

How to cite this article: da Silva TM, Fiaccone RL, Kehdy FSG, et al. African biogeographical ancestry, atopic and nonatopic asthma and atopy: A study in Latin American children. Pediatric Pulmonology. 2019;54:125-132.

https://doi.org/10.1002/ppul.24213 